\title{
CRESCIMENTO SOMÁTICO E DESEMPENHO MOTOR DE CRIANÇAS MADEIRENSES DO PRÉ-ESCOLAR
}

GROWTH AND MOTOR PERFORMANCE OF MADEIRA ISLAND PRE-SCHOOL CHILDREN

CRECIMIENTO SOMÁTICO Y RENDIMIENTO MOTOR DE NIÑOS MADERENSES DEL PREESCOLAR

António Manuel Antunes (amantunes@uma.pt) * | **

Rui Sousa (quintas.ruicsmaritimo@gmail.com)***

Rui Osório (rui.osorio@csmaritimo.pt)***

Sérgio Lopes (bessahugo@hotmail.com)***

Hugo Bessa (rui.osorio@csmaritimo.pt)***

Bernardo Valério (bernardolage.valerio@gmail.com) ${ }^{\star *}$

João Estanqueiro (estanqueirojm@gmail.com)**

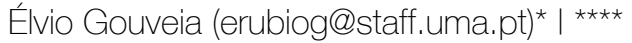

Gonçalo Marques (goncalo.marques@staff.uma.pt)* ${ }^{* *}$

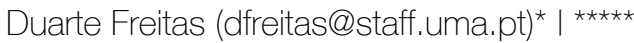

\section{RESUMO}

O objetivo foi investigar o dimorfismo sexual, a estabilidade e a mudança no crescimento somático e desempenho motor em crianças dos 3 aos 5 anos.

A amostra foi composta por 55 (23 raparigas e 32 rapazes) alunos do Colégio do Marítimo. O crescimento, através do peso, altura e perímetro de cintura, foi aferido seguindo o protocolo descrito no The Leuven Growth Study. O desempenho motor foi avaliado através da Preschool Test Battery. A avaliação realizou-se em 2 anos letivos consecutivos. O teste Mann-Whitney, a correlação de Spearman e o Wilcoxon signed rank test foram utilizados na análise.

A altura revelou diferenças $(U=230,0 ; W=819,0 ; p=0,03)$ entre meninas $(\mathrm{Md}=107,70)$ e meninos $(\mathrm{Md}=104,40)$ no $1^{\circ}$ ano, bem como um ano mais tarde. As meninas apresentaram valores medianos estaturais ( $\mathrm{Md}=112,10)$ superiores aos meninos ( $\mathrm{Md}=108,10)$. No desempenho motor, observaramse diferenças com significado estatístico no lançamento da bola de ténis nos dois momentos de avaliação. Os meninos ( $\mathrm{Md}=5,01)$ lançaram distâncias superiores às meninas $(\mathrm{Md}=3,79)$, nos $1^{\circ}$ e $2^{\circ}$ momentos de avaliação $(\mathrm{Md}=$ 
5,74 e $\mathrm{Md}=4,71$, respetivamente). A altura, nas raparigas, foi a variável que revelou maior estabilidade da $1^{a}$ para a $2^{a}$ avaliação (rho= 0,98). No desempenho motor, as correlações mais elevadas foram observadas na corrida de ida-e-volta para as meninas (rho> 0,85) e na corrida de velocidade para os meninos (rho> 0,83). A maior expressão na mudança foi observada no peso corporal, nas meninas $(Z=-2,37 ; p=0,018)$. Os scores medianos aumentaram dos $3(M d=15,40)$ para os 4 anos $(M d=17,40)$. Resultados similares foram observados na altura. No desempenho motor foram observadas melhorias estatisticamente significativas na quase totalidade dos testes. Exceções foram observadas na impulsão horizontal $(Z=-1,86, p=0,077$; meninas), corrida de velocidade $(Z=-1,83, p=0,068$; meninos) e lançamento da bola de ténis $(Z=$ $-1,85, p=0,064$; meninos).

As diferenças entre sexos no crescimento somático e desempenho motor estão presentes em idade pré-escolar. A estabilidade foi elevada nas variáveis somáticas e melhorias no desempenho motor foram mais evidentes dos 4 para os 5 anos.

\section{Palavras-chave: crianças, crescimento, performance}

\section{ABSTRACT}

The aim of this study was to investigate sexual dimorphism, stability and change in somatic growth and motor performance in children, from 3 to 5 years.

The sample consisted of 55 students (23 girls and 32 boys) from Colégio do Marítimo. Growth, through weight, height and waist circumference, was measured following the protocol described in The Leuven Growth Study. Motor performance was assessed through Preschool Test Battery. The evaluation was carried out in 2 consecutive school years. The Mann-Whitney test, the Spearman correlation and the Wilcoxon signed rank test were used in the analysis.

The height revealed differences $(U=230,0 ; W=819,0 ; p=0,03)$ among girls $(\mathrm{Md}=107,70)$ and boys $(\mathrm{Md}=104,40)$ at the 1 st evaluation moment, as well as in the following year's evaluation. The girls had higher $(\mathrm{Md}=112,10)$ mean values than boys (Md=108,10). In motor performance, statistically significant differences were observed in the throwing of the tennis ball in the two moments of evaluation. The boys ( $\mathrm{Md}=5,01)$ launched distances higher than the girls $(\mathrm{Md}=3,79)$ in the 1 st and 2 nd evaluation $(\mathrm{Md}=5,74$ e $\mathrm{Md}=4,71$ moments 
respectively. The height in girls was the variable that showed greater stability from the 1 st to the 2nd evaluation $(r h o=0,98)$. In motor performance, the highest correlations were observed in the scramble for girls (rho> 0,85) and speed run for boys (rho> 0,83). The greatest expression in the change was observed in body weight in girls. $(Z=-2,37 ; p=0,018)$. Median scores increased from $3(M d=15,4)$ to 4 years $(M d=17,4)$. Similar results were observed at height. In motor performance, statistically significant improvements were observed in almost all tests. Exceptions have been observed in horizontal jump ( $Z=$ $-1,86, p=0,077$; girls), speed run $(Z=-1,83, p=0,068$; boys) and tennis ball throw $(Z=-1,85, p=0,064$; boys $)$.

Differences between genders in somatic growth and motor performance are present at preschool age. Stability was high in somatic variables and improvements in motor performance were more evident from 4 to 5 years.

Keywords: children, growth, performance

\section{RESUMEN}

El objetivo fue investigar el dimorfismo sexual, la estabilidad y el cambio en el crecimiento somático y desempeño motor en niños de 3 a 5 años.

La muestra fue compuesta por 55 (23 chicas y 32 chicos) alumnos del Colegio do Marítimo. El crecimiento, a través del peso, altura y perímetro de cintura, fue evaluado siguiendo el protocolo descrito en The Leuven Growth Study. E rendimiento del motor se ha evaluado a través de Preschool Test Battery. La evaluación se realizó en 2 años escolares consecutivos. La prueba de MannWhitney, la correlación de Spearman y el Wilcoxon de rango de análisis se utilizaron en el análisis.

La altura reveló diferencias $(U=230,0, W=819,0, p=0,03)$ entre niñas ( $M d=$ $107,70)$ y niños $(\mathrm{Md}=104,40)$ en el primer momento de evaluación, así como un año más tarde. Las niñas presentaron valores medianos estatura ( $\mathrm{Md}=$ $112,10)$ superiores a los niños $(\mathrm{Md}=108,10)$. En el desempeño motor, se observaron diferencias con significado estadístico en el lanzamiento de la pelota de tenis en los dos momentos de evaluación. Los niños ( $\mathrm{Md}=5,01)$ arrojaron distancias superiores a las niñas $(\mathrm{Md}=3,79)$, en los $1^{\circ}$ y $2^{\circ}$ momentos de evaluación ( $\mathrm{Md}=5,74$ y $\mathrm{Md}=4,71$, respectivamente). La altura, en las niñas, fue la variable que reveló mayor estabilidad de la $1^{a}$ para la $2^{a}$ evaluación (rho= 0,98). En el desempeño motor, las correlaciones más altas se observaron en 


\title{
Egitania
}

s c i e $n$ c i a

la carrera de ida y vuelta para las niñas (rho $>0,85)$ y en la carrera de velocidad para los niños (rho> 0,83). La mayor expresión en el cambio fue observada en el peso corporal, en las niñas $(Z=-2,37 ; p=0,018)$. Las puntuaciones medianas aumentaron de los $3(M d=15,40)$ para los 4 años $(M d=17,40)$. Se observaron resultados similares en el momento. En el desempeño motor se observaron mejoras estadísticamente significativas en la casi totalidad de las pruebas. $(Z=-1,86, p=0,077$, niñas), carrera de velocidad $(Z=-1,83, p=$ 0,068 , niños) y lanzamiento de la pelota de tenis ( $Z=-1,85, p=0,064$, niños). Las diferencias entre los sexos en el crecimiento somático y el rendimiento motor están presentes en la edad preescolar. La estabilidad fue elevada en las variables somáticas y las mejoras en el rendimiento motor fueron más evidentes de los 4 a los 5 años.

\section{Palabras clave: niños, crecimiento, rendimiento}

\author{
* Universidade da Madeira, Funchal, Portugal; \\ ** Secretaria Regional da Educação, Funchal, \\ Portugal; \\ *** Colégio do Marítimo, Funchal, Portugal; \\ **** Madeira Interactive Technologies Institute, \\ Funchal, Portugal

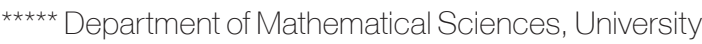 \\ of Essex, Colchester, UK
}




\section{INTRODUÇÃO}

Num Mundo onde a velocidade tecnológica cria constantes novos desafios, bem como, novas oportunidades (World Economic Forum, 2015), cada vez mais o estudo e interpretação do crescimento e desempenho motor, em especial de crianças em idades pediátricas, serão fulcrais ao combate do sedentarismo e suas enfermidades (Lou, 2014; Ridgers, Timperio, Cerin, \& Salmon, 2014). Esta responsabilidade é ainda maior quando se percebe que, com base numa revisão de literatura que abordou os últimos 30 anos, a primeira infância constitui um momento de alto relevo na promoção de comportamentos de estilos de vida saudáveis (Jones, Hinkley, Okely, \& Salmon, 2013). Nestas idades, o desenvolvimento da competência nas habilidades motoras fundamentais é um mecanismo que poderá promover, futuramente, um maior envolvimento em atividades físicas (Stodden et al., 2008) e inclusive correlações positivas em parâmetros de aptidão física associados à saúde (Robinson et al., 2015).

Embora a aptidão física tenha sido um foco central de análise de investigadores de renome internacional [ver Pediatrics Fitness: Secular Trends and Geographic Variability (Tomkinson \& Olds, 2007)], nos últimos anos, em crianças de tenra idade, as atenções têm-se centrado nas habilidades motoras (Barnett et al., 2016). Cimentada a importância, em crianças nas faixas etárias dos 3 aos 10 anos, da análise dos processos das habilidades motoras (Ulrich, 2000), é necessário e urgente perceber os atuais índices do produto motor infantil, em especial no pré-escolar. O relevo do produto ganha dimensão quando analisamos as diretrizes de atividade física para crianças. E, aqui, já tomamos como consensuais os 60 minutos de atividade diária, na condição desta integrar atividades aeróbicas intensas a vigorosas (como marcha rápida ou corrida) e de força muscular, pelo menos 3 vezes por semana (Centers for Disease Control and Prevention, 2016). É neste especial domínio que, sendo a aptidão física um estado adaptativo onde várias componentes de desempenho se relacionam com a saúde, tais como a força, flexibilidade, aptidão motora e potência aeróbica (Malina, 1996) já se percebem relações de espiral positiva com a competência motora na infância (Stodden, Gao, Goodway, \& Langendorfer, 2014), mapeadas com recuso a testes de produto. Assim, através de modelos métricos coerentes (Morris, Atwater, Williams, \& Wilmore, 1981), e de fácil inter- 
pretação para os profissionais da Educação Física, é urgente começar a mapear o desempenho motor nas crianças dos 3 aos 6 anos, algo ainda muito desconhecido, no domínio do produto, na nossa realidade educativa (Antunes, Freitas, Mendonça, \& Maia, 2013b).

É no relevo da dimensão apresentada anteriormente que, o envolvimento das escolas básicas em projetos de domínio epidemiológico, apoiadas pela comunidade científica (Centers for Disease Control and Prevention, 2010), poderá oferecer uma resposta válida à construção de novas diretrizes de combate às problemáticas do sedentarismo. Internacionalmente, são exemplo desta interdisciplinaridade o "The CHAMPS-study DK" (Wedderkopp et al., 2012) ou o estudo "MUGl" (Ericsson, 2008). Assim, programas de intervenção que dediquem uma atenção às componentes específicas do desempenho físico, ou seja, força, resistência, velocidade e agilidade, podem melhorar a probabilidade de sucesso, das crianças, no desenvolvimento dos blocos fundamentais que possibilitem a construção de um abecedário motor de qualidade, bem como viabilizar o envolvimento em atividades físicas de nível superior (Chen, Hammond-Bennett, \& Hypnar, 2017; Fidler et al., 2016).

Num compromisso de elevada responsabilidade educativa, o Colégio do Marítimo integrou um estudo piloto da Secretaria Regional de Educação, denominado "Pensar, Agir e Refletir (PAR): um caminho de sucesso para o diálogo corporal da criança" (Antunes, 2014), que se centrou na rentabilização espaciotemporal e na melhoria das dinâmicas das aulas de Educação Física, através da participação ativa e responsável dos diversos atores educativos. Desta participação, decorreu o nascimento do projeto "Super Garrinhas", tendo como missão a avaliação do crescimento físico humano e dos níveis de performance motora, das crianças do pré-escolar da organização.

A recolha de informação permitiu-nos construir os seguintes propósitos:

- analisar as diferenças entre géneros no crescimento e desempenho motor de crianças do pré-escolar;

- caracterizar a estabilidade do crescimento e da performance, entre os 3 e os 5 anos, em rapazes e raparigas, e;

- perceber a variabilidade do crescimento e da performance motora ao longo da idade. 


\section{METODOLOGIA}

\section{AMOSTRA E DELINEAMENTO}

A presente pesquisa retrata um estudo de modelo longitudinal, onde no ano letivo 2014/2015 foram avaliadas 55 crianças (23 raparigas e 32 rapazes), do pré-escolar, do Colégio do Marítimo. Todas as crianças foram reavaliadas no ano letivo posterior (2015/2016), isto é, sensivelmente um ano mais tarde (ver Tabela 1). Assim, avaliou-se um Grupo 1 constituído por 21 crianças ( 3 para os 4 anos) e um Grupo 2 composto por 34 crianças (4 para os 5 anos de idade). A totalidade dos alunos do colégio foram convidados a participar no projeto. Os Encarregados de Educação assinaram o consentimento informado para os participantes integrarem o "Super Garrinhas".

Tabela 1: Valores descritivos da idade decimal (nos dois momentos de avaliação)

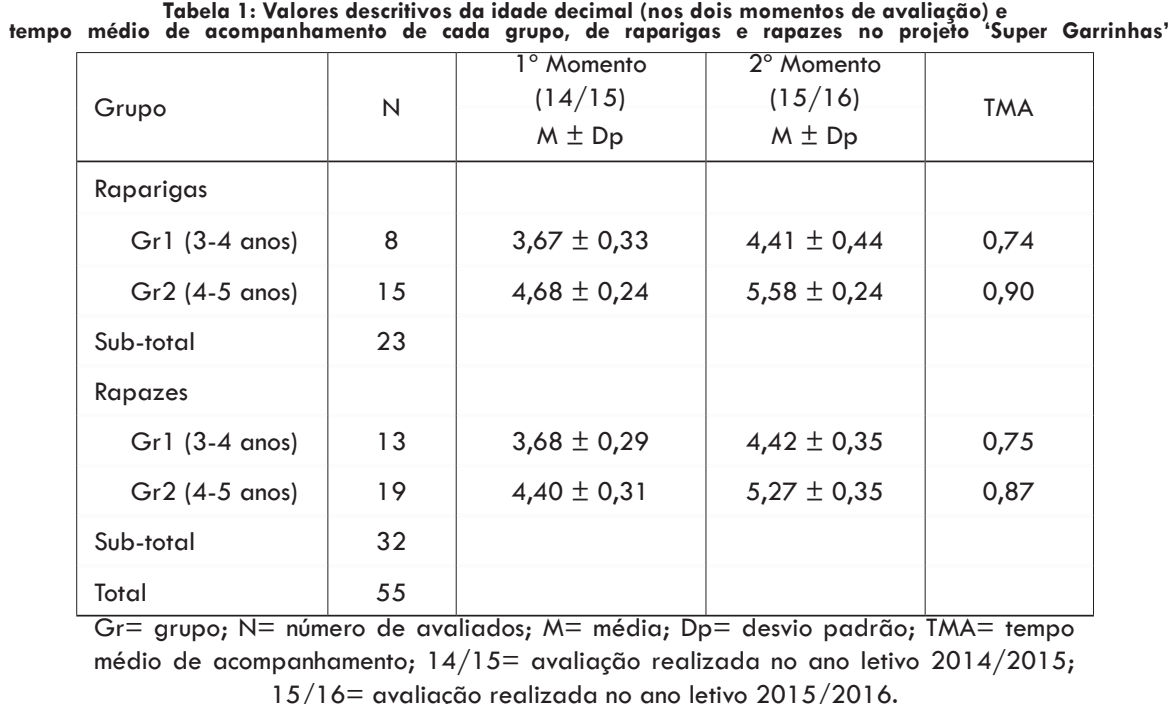

\section{CRESCIMENTO FÍSICO}

A altura, o peso corporal e o perímetro da cintura (Pcin) foram medidos de acordo com os procedimentos utilizados no The Leuven Growth 
Study e descritos, mais recentemente, por Claessens, Beunen, \& Malina (2008). O índice de massa corporal (IMC) foi aferido com recurso ao quociente entre o peso e a altura ao quadrado.

\section{DESEMPENHO MOTOR}

O desempenho motor, das crianças do pré-escolar do Colégio do Marítimo, foi avaliado através do protocolo definido por "Preschool Test Battery" (PTB) de (Morris, Atwater, Williams, \& Wilmore, 1981). A bateria inclui 6 testes, nomeadamente:

- Agarrar (Ag)- quantos pontos o avaliado consegue atingir apanhando uma bola que the foi lançada pelo avaliador;

- Impulsão Horizontal (IH)- quantos centímetros salta a pés juntos, sem corrida preparatória;

- Corrida de Ida-e-Volta (Clv)- quanto tempo demora a levantar-se da posição de decúbito dorsal, percorrer 3,047 metros, apanhar uma bola e regressar ao ponto de partida;

- Corrida de Velocidade (CV)- quanto tempo demora a percorrer a distância de 12,19 metros, após o período de aceleração de 3,66m;

- Equilibrio (Eq)- quantos segundos consegue a criança equilibrarse num bloco de madeira específico, e;

- Lançamento da Bola de Ténis (LBT)- quantos metros uma criança consegue lançar uma bola de ténis.

\section{FIABILIDADE}

A informação foi recolhida pelo coordenador de Expressão e Educação Físico Motora e por dois docentes da disciplina da escola. Os elementos da equipa de campo tiveram formação teórico prática específica, com um especialista da Universidade da Madeira, para a recolha da informação de ambos os domínios. Posteriormente, realizaram um estudo piloto onde obtiveram consistência elevada no teste-reteste. Os coeficientes de correlação intraclasse $(\mathrm{CCl})$ variaram, nas variáveis somáticas, entre 
0,97 no Pcin e 0,99 no peso e altura. No que concerne aos testes da PTB, os CCl balizaram-se entre 0,79 e 0,96 nas provas do Ag e CV, respetivamente (Sousa et al., 2016).

\section{PROCEDIMENTOS ESTATÍSTICOS}

Todos os cálculos foram efetuados com recurso ao Statistical Package for Social Sciences, versão 23,0. A informatização dos dados operacionalizou-se através do processo de dupla entrada, com recurso à ferramenta 'Form' da Google Docs baseado em Asynchronous Javascript and Extensible Markup Language. Realizou-se análise exploratória eliminando-se os outliers. A análise de dados iniciou-se com a caracterização da amostra através de tabelas de frequências. As medidas descritivas usadas foram a média (M), a mediana (Md) e o desvio padrão (Dp). As diferenças entre meninas e meninos foram aferidas com recurso ao teste Wilcoxon-Mann-Whitney U. A estabilidade, nas variáveis do crescimento somático e no desempenho motor, para rapazes e raparigas, foi calculada através do coeficiente de correção produto-momento de Spearman. A variação ao longo da idade foi analisada utilizando-se o Wilcoxon Signed Rank Test para medidas emparelhadas. O nível de significância foi mantido em 5\%.

\section{RESULTADOS}

\section{DIMORFISMO SEXUAL}

As diferenças entre raparigas e rapazes foram avaliadas no $1 .^{\circ}$ (ano letivo 14/15) e 2. (ano letivo 15/16) momentos de avaliação. Os valores estão reportados na Tabela 2.

Tabela 2: Diferenças entre sexos, do crescimento e do desempenho motor, nos diferentes momentos de avaliação.

\begin{tabular}{|c|c|c|c|c|c|c|c|c|}
\hline \multirow{2}{*}{ Variáveis } & \multicolumn{2}{|c|}{ Fem } & \multicolumn{2}{|c|}{ Masc } & \multirow{2}{*}{ U } & \multirow{2}{*}{ W } & \multirow{2}{*}{$p$} & \multirow{2}{*}{$r$} \\
\hline & $\mathrm{N}$ & Md & $\mathrm{N}$ & Md & & & & \\
\hline
\end{tabular}




\begin{tabular}{|c|c|c|c|c|c|c|c|c|}
\hline \multicolumn{9}{|c|}{$1^{\circ}$ Momento de avaliação $(14 / 15)$} \\
\hline \multicolumn{9}{|l|}{$\begin{array}{l}\text { Crescimento } \\
\text { somático }\end{array}$} \\
\hline Peso & 21 & 17,60 & 32 & 16,25 & 264,5 & 792,5 & 0,19 & 0,18 \\
\hline Altura & 23 & 107,70 & 31 & 104,40 & 230,0 & 726,0 & 0,03 & 0,30 \\
\hline IMC & 21 & 15,74 & 31 & 15,42 & 323,0 & 819,0 & 0,96 & 0,01 \\
\hline Pcin & 21 & 52,40 & 32 & 51,70 & 330,5 & 858,5 & 0,92 & 0,01 \\
\hline \multicolumn{9}{|l|}{$\begin{array}{l}\text { Desempenho } \\
\text { motor }\end{array}$} \\
\hline $\mathrm{Ag}$ & 23 & 1,80 & 32 & 1,90 & 333,0 & 609,0 & 0,55 & 0,08 \\
\hline $\mathrm{IH}$ & 23 & 72,00 & 32 & 78,50 & 322,5 & 598,5 & 0,44 & 0,11 \\
\hline $\mathrm{Clv}$ & 22 & 5,25 & 32 & 5,32 & 338,0 & 591,0 & 0,81 & 0,03 \\
\hline $\mathrm{CV}$ & 22 & 3,75 & 32 & 3,69 & 333,5 & 861,5 & 0,75 & 0,04 \\
\hline Eq & 21 & 8,00 & 28 & 7,00 & 263,5 & 669,5 & 0,54 & 0,09 \\
\hline LBT & 18 & 3,79 & 31 & 5,01 & 141,5 & 312,5 & $<0,001$ & 0,41 \\
\hline \multicolumn{9}{|c|}{$2^{\circ}$ Momento de avaliação $(15 / 16)$} \\
\hline \multicolumn{9}{|l|}{$\begin{array}{l}\text { Crescimento } \\
\text { somático }\end{array}$} \\
\hline Peso & 21 & 19,60 & 32 & 18,30 & 278,0 & 806,0 & 0,29 & 0,15 \\
\hline Altura & 23 & 112,10 & 31 & 108,10 & 222,5 & 718,5 & 0,02 & 0,32 \\
\hline IMC & 21 & 15,91 & 31 & 15,40 & 300,0 & 796,0 & 0,63 & 0,07 \\
\hline Pcin & 19 & 51,80 & 32 & 52,75 & 251,5 & 441,5 & 0,31 & 0,14 \\
\hline \multicolumn{9}{|l|}{$\begin{array}{l}\text { Desempenho } \\
\text { motor }\end{array}$} \\
\hline $\mathrm{Ag}$ & 23 & 2,60 & 32 & 2,50 & 346,0 & 622,0 & 0,71 & 0,05 \\
\hline $\mathrm{IH}$ & 18 & 94,50 & 32 & 96,00 & 284,0 & 812,0 & 0,94 & 0,01 \\
\hline $\mathrm{Clv}$ & 22 & 4,68 & 31 & 4,66 & 326,5 & 822,5 & 0,79 & 0,04 \\
\hline $\mathrm{CV}$ & 18 & 3,19 & 32 & 3,27 & 247,5 & 418,5 & 0,41 & 0,12 \\
\hline $\mathrm{Eq}$ & 22 & 19,60 & 27 & 16,80 & 229,0 & 607,0 & 0,17 & 0,20 \\
\hline LBT & 20 & 4,71 & 32 & 5,74 & 181,0 & 391,0 & 0,01 & 0,36 \\
\hline
\end{tabular}

$\mathrm{N}$ : número de avaliados; Md: mediana; U: estatística de Mann-Whitney; W: estatística de Wilcoxon; $\mathrm{p}$ : probabilidade de significância; r: tamanho do efeito estatístico

O teste Mann-Whitney U revelou não existirem diferenças significativas (p>0,05), nos dois momentos de avaliação, entre raparigas e rapazes no peso, no IMC e no Pcin. Estes dados corroboram com outros estudos, com informações recolhidas em 2400 crianças do concelho de Coimbra (Rito, 2004) e em 836 crianças em idade escolar da Região Autónoma da Madeira do pré-escolar e primeiro ciclo (Antunes, Freitas, 
Mendonça, \& Maia, 2013a), onde as diferenças entre sexos apresentam expressão reduzida.

Contudo, a altura revelou-se estatisticamente diferente $(U=230,0 ; W=$ 819,0; $\mathrm{p}=$ 0,03) entre meninas ( $\mathrm{Md}=107,70)$ e meninos $(\mathrm{Md}=104,40)$ no ano letivo 14/15. Esta diferença manteve-se significativa $(U=222,5$; $\mathrm{W}=718,5 ; \mathrm{p}=0,02) \mathrm{um}$ ano mais tarde, onde as meninas apresentam valores medianos da estatura $(\mathrm{Md}=112,10)$ superiores aos meninos $(\mathrm{Md}=108,10)$.

No que concerne ao desempenho motor, apenas se observaram diferenças com significado estatístico na prova do LBT no 1. ${ }^{\circ}(U=141,5$; $W=312,5 ; p<0,001)$ e 2. ${ }^{\circ}(U=181,0 ; W=391,0 ; p=0,01)$ momentos de avaliação. Assim, no ano letivo 14/15 aos rapazes ( $\mathrm{Md}=5,01$ ) lançaram distâncias maiores relativamente às colegas ( $\mathrm{Md}=3,79)$, bem como, no ano letivo 15/16 ( $\mathrm{Md}=5,74$ e $\mathrm{Md}=4,71$, respetivamente).

No padrão de desempenho motor, delineado em crianças norte-americanas por (Morris, Williams, Atwater, \& Wilmore, 1982), verificou-se que o sexo foi um fator mais preponderante do que a idade no LBT, com benefício para os meninos. Antunes et al. (2013b) constatou, igualmente, que os rapazes lançavam distâncias médias maiores do que as raparigas, confirmando os resultados obtidos pelas crianças do Colégio do Marítimo. A correlação positiva entre controlo de objetos e o sexo masculino está também confirmada, em revisões bibliográficas de desempenho motor infantil, nos domínios das habilidades motoras fundamentais (livonen \& Sääkslahti, 2014) e da coordenação motora (Barnett et al., 2016).

\section{ESTABILIDADE E VARIABILIDADE NO CRESCIMENTO E DESEMPENHO MOTOR}

A estabilidade nas variáveis do crescimento somático, bem como no desempenho motor, para rapazes e raparigas, foi aferida através do coeficiente de correlação produto-momento de Spearman, como se pode verificar na Tabela 3. 


\begin{tabular}{|c|c|c|c|c|}
\hline \multirow{2}{*}{ Variáveis } & \multicolumn{2}{|c|}{ Feminino } & \multicolumn{2}{|c|}{ Masculino } \\
\hline & rho & $p$ & rho & $p$ \\
\hline \multicolumn{5}{|l|}{ Crescimento Físico Humano } \\
\hline Peso &, $917^{* *}$ & $<, 001$ &, $928 * *$ & $<, 001$ \\
\hline Altura &, $980 * *$ & $<, 001$ &, $917^{* *}$ & $<, 001$ \\
\hline $\begin{array}{l}\text { Índice de massa } \\
\text { corporal (IMC) }\end{array}$ &, $652 * *$ & 001 &, $779 * *$ & $<, 001$ \\
\hline Perímetro da cintura (Pcin) &, $743^{* *}$ & $<, 001$ &, $803 * *$ & $<, 001$ \\
\hline \multicolumn{5}{|l|}{ Desempenho Motor (PTB) } \\
\hline Agarrar $(\mathrm{Ag})$ &, $604^{* *}$ &, 002 &, $701 * *$ & $<, 001$ \\
\hline Impulsão horizontal (IH) & 400 & 104 &, $802 * *$ & $<, 001$ \\
\hline Corrida ida-e-volta (Clv) &, $848 * *$ & $<, 001$ &, $723 * *$ & $<, 001$ \\
\hline $\begin{array}{l}\text { Corrida de velocidade } \\
\text { (CV) }\end{array}$ &, $717^{* *}$ & 001 &, $825^{* *}$ & $<, 001$ \\
\hline Equilíbrio (Eq) &, $747^{* *}$ & $<, 001$ & $454^{*}$ & 020 \\
\hline $\begin{array}{l}\text { Lançamento da bola de } \\
\text { ténis (LBT) }\end{array}$ &, $512^{*}$ &, 036 &, $750 * *$ & $<, 001$ \\
\hline
\end{tabular}

rho $=$ Coeficiente de correlação de Spearman; *nível de significância 0.05 (bilateral); ** nível de significância 0.01 (bilateral), $p=$ probabilidade de significância

Existe uma forte correlação positiva, entre todas as variáveis de crescimento físico humano, do 1. ${ }^{\circ}$ para o 2. ${ }^{\circ}$ momento de avaliação, onde o valor mínimo se verifica no $\mathrm{IMC}(\mathrm{rho}=0,652, \mathrm{p}=0,001)$ e o máximo na altura ( $r h o=0,980, p<0,001$ ), ambos nas raparigas. Relativamente ao desempenho motor, não encontrámos significado na correlação, das meninas, no teste da $H$ (rho> $0,400, p=0,104)$. A relação mais baixa revelou-se nos meninos, no teste do Eq (rho> 0,454, $\mathrm{p}=0,020$ ) de magnitude moderada. Todos os restantes testes, da PTB, revelaram fortes correlações entre os dois momentos de avaliação. A maior similitude, nas raparigas constatou-se na prova da Clv (rho> 0,848, $p<$ 0,001), nos rapazes ocorreu no teste da CV (rho $>0,825, p<0,001$ ).

A variação ao longo da idade (nos dois grupos em análise) foi aferida através do Wilcoxon Signed Rank Test para medidas emparelhadas, para raparigas (ver tabela 4a) e rapazes (ver tabela 4b) separadamente, nos indicadores somáticos e nas provas de desempenho motor.

No âmbito do crescimento, a altura e o peso revelaram incrementos significativos ao longo da idade nos dois grupos $(p<0,05)$. No peso a 
maior expressão ocorreu nas meninas, $Z=-2,37, p=0,018$, com largo efeito $(r=0,63)$, onde os scores medianos aumentam dos $3(\mathrm{Md}=15,40)$ para os 4 anos ( $\mathrm{Md}=17,40)$. Na altura, os maiores ganhos revelaramse, igualmente, nas meninas do Gr1, $Z=-2,52, p=0,012$, com largo efeito $(r=0,63)$, onde os scores medianos aumentam aproximadamente $5 \mathrm{~cm}$. As diferenças entre os valores medianos do IMC e do Pcin (do 1.' para o 2.' momento de avaliação) não revelaram significado estatístico entre raparigas e rapazes (p> 0,05) em ambos os grupos etários. Relativamente ao desempenho motor, em todos os testes da PTB, existiram melhorias significativas nas performances ao longo da idade, em ambos os sexos, com elevada expressão no Gr2 (dos 4 para os 5 anos), bem como globalmente no Gr1 (dos 3 para os 4 anos). No entanto, os resultados medianos das meninas, no Gr1, não revelaram diferenças com significado estatístico no teste da $\|(Z=-1,86$, $\mathrm{p}=0,077)$ e na prova de $\mathrm{CV}(\mathrm{Z}=-1,83, \mathrm{p}=0,068)$. Nos rapazes, $\mathrm{no}$ teste LBT, dos $3(\mathrm{Md}=4,44)$ para os 4 anos $(\mathrm{Md}=5,14)$, o aumento do desempenho, também não se revelou estatisticamente significativo $(Z=$ $-1,85, p=0,064)$.

Tabela 4a: Variação do crescimento e do desempenho motor, ao longo da idade, para raparigas.

\begin{tabular}{|c|c|c|c|c|c|c|c|}
\hline \multirow{2}{*}{ Variáveis } & \multirow{2}{*}{$N$} & \multicolumn{2}{|c|}{$1^{\circ}$ Momento } & \multicolumn{2}{|c|}{$2^{\circ}$ Momento } & \multirow{2}{*}{$z$} & \multirow{2}{*}{$p$} \\
\hline & & $M \pm D p$ & Med & $M \pm D p$ & Med & & \\
\hline \multicolumn{8}{|c|}{$\begin{array}{l}\text { Gr } 1 \quad(3-4 \\
\text { anos) }\end{array}$} \\
\hline \multicolumn{8}{|c|}{ Crescimento somático } \\
\hline Peso & 7 & $15,53 \pm 1,47$ & 15,4 & $17,47 \pm 1,63$ & 17,4 & $-2,37$ & 0,018 \\
\hline Altura & 8 & $101,36 \pm 5,34$ & 101,75 & $106,96 \pm 4,85$ & 106,7 & $-2,52$ & 0,012 \\
\hline IMC & 7 & $15,47 \pm 0,98$ & 14,9 & $15,65 \pm 1,01$ & 15,2 & $-0,68$ & 0,499 \\
\hline Pcin & 7 & $50,57 \pm 2,49$ & 51,3 & $51,26 \pm 1,86$ & 51,4 & $-0,68$ & 0,496 \\
\hline \multicolumn{8}{|c|}{$\begin{array}{l}\text { Desempenho } \\
\text { motor }\end{array}$} \\
\hline $\mathrm{Ag}$ & 8 & $1,66 \pm 0,36$ & 1,65 & $2,08 \pm 0,52$ & 2 & $-2,03$ & 0,043 \\
\hline $\mathrm{IH}$ & 8 & $49,38 \pm 23,96$ & 40 & $96,5 \pm 8,23$ & 98 & $-1,86$ & 0,077 \\
\hline $\mathrm{Clv}$ & 7 & $5,88 \pm 0,7$ & 6,13 & $5,23 \pm 0,59$ & 5,32 & $-2,37$ & 0,018 \\
\hline$C V$ & 7 & $4,02 \pm 0,6$ & 3,88 & $3,27 \pm 0,21$ & 3,23 & $-1,83$ & 0,068 \\
\hline Eq & 7 & $3,71 \pm 2,43$ & 4 & $15,63 \pm 12,12$ & 14,3 & $-2,37$ & 0,018 \\
\hline LBT & 6 & $3,73 \pm 0,65$ & 3,79 & $4,34 \pm 1,09$ & 4,4 & $-2,2$ & 0,028 \\
\hline
\end{tabular}




\begin{tabular}{|c|c|c|c|c|c|c|c|}
\hline $\begin{array}{l}\text { Gr2 } \\
\text { anos) }\end{array}$ & & & & & & & \\
\hline \multicolumn{8}{|c|}{ Crescimento somático } \\
\hline Peso & 14 & $18,7 \pm 1,73$ & 19,1 & $20,59 \pm 2,22$ & 20,8 & $-3,24$ & 0,001 \\
\hline Altura & 15 & $110,13 \pm 3,89$ & 110,4 & $113,96 \pm 3,9$ & 113,6 & $-3,41$ & 0,001 \\
\hline IMC & 14 & $15,53 \pm 0,88$ & 15,77 & $15,96 \pm 0,92$ & 16,11 & $-1,66$ & 0,096 \\
\hline Pcin & 14 & $52,45 \pm 2,48$ & 52,5 & $52,23 \pm 1,89$ & 52,25 & $-0,63$ & 0,53 \\
\hline \multicolumn{8}{|c|}{$\begin{array}{l}\text { Desempenho } \\
\text { motor }\end{array}$} \\
\hline $\mathrm{Ag}$ & 15 & $1,99 \pm 0,45$ & 1,8 & $2,55 \pm 0,31$ & 2,7 & $-3,24$ & 0,001 \\
\hline $\mathrm{IH}$ & 15 & $80,2 \pm 13,64$ & 80 & $96 \pm 6,03$ & 94,5 & $-3,2$ & 0,001 \\
\hline $\mathrm{Clv}$ & 15 & $5,11 \pm 0,58$ & 5,17 & $4,53 \pm 0,3$ & 4,54 & $-3,41$ & 0,001 \\
\hline $\mathrm{CV}$ & 15 & $3,67 \pm 0,39$ & 3,57 & $3,15 \pm 0,17$ & 3,19 & $-3,3$ & 0,001 \\
\hline $\mathrm{Eq}$ & 14 & $14,93 \pm 8,85$ & 11,5 & $26,33 \pm 12,25$ & 28,8 & $-3,3$ & 0,001 \\
\hline LBT & 12 & $3,8 \pm 0,57$ & 3,79 & $4,85 \pm 0,81$ & 4,81 & $-2,85$ & 0,004 \\
\hline
\end{tabular}

Gr: grupo; N: número de avaliados; $M$ : média; Dp: desvio padrão; $M d$ : mediana; Z: Wilcoxon Signed Rank Test; p: probabilidade de significância

\begin{tabular}{|c|c|c|c|c|c|c|c|}
\hline \multicolumn{8}{|c|}{ Tabela 4b: Variação do crescimento e do desempenho motor, ao longo da idade, para rapazes. } \\
\hline \multirow[b]{2}{*}{ Variáveis } & \multirow{2}{*}{$\mathrm{N}$} & \multicolumn{2}{|c|}{$1^{\circ}$ Momento } & \multicolumn{2}{|c|}{$2^{\circ}$ Momento } & \multirow{2}{*}{ z } & \multirow{2}{*}{$p$} \\
\hline & & $M \pm D p$ & Med & $M \pm D p$ & Med & & \\
\hline \multicolumn{8}{|l|}{ Gr1 (3-4 anos) } \\
\hline \multicolumn{8}{|c|}{ Crescimento somático } \\
\hline Peso & 13 & $16,13 \pm 1,55$ & 16,2 & $18,15 \pm 2,29$ & 17,5 & $-3,18$ & 0,001 \\
\hline Altura & 13 & $99,98 \pm 4,16$ & 98,4 & $105,14 \pm 4,19$ & 103,3 & $-3,19$ & 0,001 \\
\hline IMC & 12 & $15,84 \pm 0,99$ & 15,99 & $16,01 \pm 0,98$ & 15,96 & $-1,18$ & 0,239 \\
\hline Pcin & 13 & $51,82 \pm 2,51$ & 52,2 & $52,88 \pm 2,9$ & 52,9 & $-1,75$ & 0,081 \\
\hline \multicolumn{8}{|l|}{$\begin{array}{l}\text { Desempenho } \\
\text { motor }\end{array}$} \\
\hline $\mathrm{Ag}$ & 13 & $1,93 \pm 0,4$ & 1,9 & $2,37 \pm 0,46$ & 2,4 & $-3,05$ & 0,002 \\
\hline $\mathrm{IH}$ & 13 & $59,77 \pm 18,95$ & 56 & $85,23 \pm 15,47$ & 80 & $-3,18$ & 0,001 \\
\hline $\mathrm{Clv}$ & 13 & $5,7 \pm 0,5$ & 5,51 & $4,86 \pm 0,5$ & 4,83 & $-3,18$ & 0,001 \\
\hline $\mathrm{CV}$ & 13 & $4,06 \pm 0,37$ & 4,21 & $3,42 \pm 0,25$ & 3,45 & $-3,18$ & 0,001 \\
\hline $\mathrm{Eq}$ & 12 & $6,67 \pm 3,89$ & 6,0 & $15,38 \pm 3,41$ & 15,2 & $-3,06$ & 0,002 \\
\hline LBT & 13 & $4,41 \pm 1,22$ & 4,44 & $5,03 \pm 1,23$ & 5,14 & $-1,85$ & 0,064 \\
\hline Gr2 (4-5 anos) & & & & & & & \\
\hline
\end{tabular}




\begin{tabular}{|l|c|c|c|c|c|c|c|}
\hline Peso & 19 & $17,48 \pm 1,95$ & 17,3 & $19,44 \pm 2,42$ & 18,5 & $-3,83$ & $<0,001$ \\
\hline Altura & 18 & $106,34 \pm 4,27$ & 106,55 & $110,51 \pm 3,16$ & 109,9 & $-3,73$ & $<0,001$ \\
\hline IMC & 19 & $15,2 \pm 1,27$ & 15,31 & $15,61 \pm 1,13$ & 15,33 & $-2,25$ & 0,242 \\
\hline Pcin & 19 & $51,83 \pm 2,78$ & 51,7 & $52,97 \pm 2,93$ & 51,6 & $-2,96$ & 0,308 \\
\hline $\begin{array}{l}\text { Dese mpenho } \\
\text { motor }\end{array}$ & & & & & & \\
\hline Ag & 19 & $1,98 \pm 0,57$ & 1,9 & $2,52 \pm 0,33$ & 2,5 & $-3,60$ & $<0,001$ \\
\hline IH & 19 & $86,21 \pm 17,01$ & 84 & $101,58 \pm 14,96$ & 103 & $-3,83$ & $<0,001$ \\
\hline Clv & 19 & $5,17 \pm 0,74$ & 5,16 & $4,58 \pm 0,47$ & 4,49 & $-3,59$ & $<0,001$ \\
\hline CV & 19 & $3,52 \pm 0,41$ & 3,42 & $3,11 \pm 0,25$ & 3,05 & $-3,73$ & $<0,001$ \\
\hline Eq & 16 & $9,13 \pm 3,54$ & 9,5 & $18,33 \pm 5,62$ & 17,8 & $-3,23$ & 0,001 \\
\hline LBT & 18 & $5,53 \pm 1,64$ & 5,52 & $7,22 \pm 2,49$ & 7,74 & $-3,40$ & 0,001 \\
\hline
\end{tabular}

Gr: grupo; N: número de avaliados; M: média; Dp: desvio padrão; Md: mediana; Z: Wilcoxon Signed Rank Test; p: probabilidade de significância

Os nossos resultados confirmam os incrementos ponderais nas variáveis somáticas ao longo da idade, dos 3 para os 5 anos, bem retratado em literatura nacional (Antunes et al., 2013a; Rito, 2004) e internacional (World Health Organization, 2006). No desempenho motor obtivemos correlações médias a elevadas similares aos valores apresentados por Rudd et al. (2015) e por Stodden et al. (2014) em habilidades de locomoção e manipulação, bem como, em habilidades coordenativas (Ahnert, Schneider, \& Bös, 2009; D'Hondt et al., 2014). Estas informações dão enfase à necessidade do desenvolvimento das capacidades condicionais e coordenativas em idade pré-escolar (Chen et al., 2017), sendo necessária uma forte aposta num currículo educativo que desenvolva e potencialize as capacidades dos alunos nestes domínios.

\section{CONCLUSÃO}

Nas características somáticas, as diferenças na altura, entre meninos e meninas, mantiveram-se consistentes e significativas nos dois momentos de avaliação. O peso, IMC e o Pcin não revelaram diferenças significativas entre os géneros. Os rapazes obtiveram performances 
superiores no LBT relativamente às meninas. Existiu estabilidade nas variáveis somáticas e nos testes motores, em rapazes e raparigas, do pré-escolar. As crianças aumentaram de peso e altura da 1. a para a 2.a avaliação. As performances melhoraram ao longo da idade, sendo que dos 4 para os 5 anos revelam maior expressão.

Para finalizar, sendo o número total de participantes uma limitação, seria importante alargar a recolha de informação longitudinal a outras escolas do pré-escolar da Região. Por outro lado, o acompanhamento destes participantes no 1. Ciclo do Ensino Básico, possibilitaria a interpretação continua os índices de crescimento e das performances motoras e, simultaneamente, a identificação de possíveis preditores desses resultados.

\section{BIBLIOGRAFIA}

Ahnert, J., Schneider, W., \& Bös, K. (2009). Developmental Changes and Individual Stability of Motor Abilities from the Preschool Period to Young Adulthood. In W. Schneider \& M. Bullock (Eds.), Human development from early childhood to early adulthood: Evidence from the Munich Longitudinal Study on the Genesis of Individual Competencies (LOGIC) (pp. 45-79). Mahwah, NJ: Erlbaum.

Antunes, A. M. (2014). Pensar, Agir e Refletir (PAR): Um caminho de sucesso para o diálogo corporal da criança. (1). Secretaria Regional da Educação e Recursos Humanos.

Antunes, A. M., Freitas, D. L., Mendonça, S. M., \& Maia, J. A. (2013a). Altura, peso corporal, altura sentado, índice de massa corporal e prevalência do sobrepeso e da obesidade em crianças madeirenses com idades compreendidas entre os 3 e os 10 anos. In J. A. Maia, F. J. Fernandes, \& D. L. Freitas (Eds.), Crescer com Saúde na Região Autónoma da Madeira. Funchal, Portugal: Universidade da Madeira \& Universidade do Porto.

Antunes, A. M., Freitas, D. L., Mendonça, S. M., \& Maia, J. A. (2013b). Desempenho motor, gordura corporal e atividade física. Um estudo em crianças madeirenses dos 3 aos 6 anos. In J. A. Maia, F. J. Fernandes, \& D. L. Freitas (Eds.), Crescer com Saúde na Região Autónoma da Madeira (pp. 503). Funchal, Portugal: Universidade da Madeira \& Universidade do Porto.

Barnett, L. M., Lai, S. K., Veldman, S. L., Hardy, L. L., Cliff, D. P., Morgan, P. J., . . Okely, A. D. (2016). Correlates of Gross Motor Competence in Children and Adolescents: A Systematic Review and Meta-Analysis. Sports Medicine, 46(11), 1663-1688. doi: 10.1007/s40279-016-0495-z 


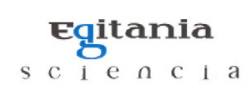

Centers for Disease Control and Prevention. (2010). The association between school based physical activity, including physical education, and academic performance. Atlanta, GA: U.S. Department of Health and Human Services.

Centers for Disease Control and Prevention. (2016). How much physical activity do children need? ,from https://www.cdc.gov/physicalactivity/basics/children/ Chen, W., Hammond-Bennett, A., \& Hypnar, A. (2017). Examination of motor skill competency in students: evidence-based physical education curriculum. BMC Public Health, 17(1), 222. doi: 10.1 186/s 1 2889-017-4105-2

Claessens, A. L., Beunen, G., \& Malina, R. M. (2008). Anthropometry, physique, body composition and maturity. In N. Armstrong \& W. van Mechelen (Eds.), Paediatric Exercise Science and Medicine (2 ed., pp. 23-36). United Kingdom- Oxford: Oxford University Press.

D'Hondt, E., Deforche, B., Gentier, I., Verstuyf, J., Vaeyens, R., De Bourdeaudhuii, I., .. . Lenoir, M. (2014). A longitudinal study of gross motor coordination and weight status in children. Obesity (Silver Spring), 22(6), 1505-1511. doi: 10.1002 /oby. 20723

Ericsson, I. (2008). Motor skills, attention and academic achievements. An intervention study in school years 1-3. British Educational Research Journal, 34(3), 301-313. doi: 10.1080/01411920701609299

Fidler, J., McLaughlin, P., Bubela, D., Scarneo, S. E., McGarry, J., Evanovich, J., \& DiStefano, L. (2016). An Exploration of the Relationship of Body Mass Index with Motor Performance Measures and Quality of Life in Children Living in an Urban Setting. J Child Obes, 1 (4), 20-26. doi: 10.21767/2572-5394.10020

livonen, S., \& Sääkslahti, A. K. (2014). Preschool children's fundamental motor skills: a review of significant determinants. Early Child Development and Care, 184(7), $1107-1126$. doi: 10.1080/03004430.2013.837897

Jones, R. A., Hinkley, T., Okely, A. D., \& Salmon, J. (2013). Tracking physical activity and sedentary behavior in childhood: a systematic review. Am J Prev Med, 44(6), 651-658. doi: 10.1016/i.amepre.2013.03.001

Lou, D. (2014). Sedentary Behaviors and Youth: Current Trends and the Impact on Health CA: Active Living Research. California, San Diego: Robert Wood Johnson Foundation.

Malina, R. M. (1996). Tracking of physical activity and physical fitness across the lifespan. Research Quarterly for Exercise and Sport, 67(3), 48-57.

Morris, A. M., Atwater, A. E., Williams, J. M., \& Wilmore, J. H. (1981). Motor performance and anthropometric screening: measurements for preschool age children. In A. M. Morris (Ed.), Motor Development: Theory into Practice (pp. 4964). Motor Skills: Theory Into Practice: Monograph 3.

Morris, A. M., Williams, J. M., Atwater, A. E., \& Wilmore, J. H. (1982). Age and Sex Differences in Motor Performance of 3 through 6 Year Old Children. Re- 
search Quarterly for Exercise and Sport, 53(3), 21 4-221.

Ridgers, N. D., Timperio, A., Cerin, E., \& Salmon, J. (2014). Compensation of physical activity and sedentary time in primary school children. Medicine and Science in Sports and Exercise, 46(8), 1564-1569. doi: 10.1249/ MSS.0000000000000275

Rito, A. I. G. (2004). Estado nutricional de crianças e oferta alimentar do pré-escolar do município de Coimbra, Portugal, 2001. (Doctoral Thesis), Fundação Oswaldo Cruz, Rio de Janeiro, Brasil.

Robinson, L. E., Stodden, D. F., Barnett, L. M., Lopes, V. P., Logan, S. W., Rodrigues, L. P., \& D'Hondt, E. (2015). Motor Competence and its Effect on Positive Developmental Trajectories of Health. Sports Medicine, 45(9), $1273-1284$. doi: 10.1007/s40279-015-0351-6

Rudd, J. R., Barnett, L. M., Butson, M. L., Farrow, D., Berry, J., \& Polman, R. C. J. (2015). Fundamental Movement Skills Are More than Run, Throw and Catch: The Role of Stability Skills. PloS One, 10(10), 15. doi: e0140224. doi:10.1371/ journal.pone.0140224

Sousa, R., Osório, R., Lopes, S., Bessa, H., Freitas, D., Maia, J., . . Antunes, A. (2016). Crescimento e desempenho motor em crianças do pré-escolar: resultados preliminares de um projeto educativo implementado no Colégio do Marítimo. In Universidade da Madeira (Ed.), Seminário Desporto e Ciência 2016. Funchal, Portugal.

Stodden, D. F., Gao, Z., Goodway, J. D., \& Langendorfer, S. J. (2014). Dynamic relationships between motor skill competence and health-related fitness in youth. Pediatric Exercise Science, 26(3), 231 -241. doi: 10.1 123/pes.2013-0027

Stodden, D. F., Goodway, J. D., Langendorfer, S. J., Roberton, M. A., Rudisill, M. E., Garcia, C., \& Garcia, L. E. (2008). A Developmental Perspective on the Role of Motor Skill Competence in Physical Activity: An Emergent Relationship. Quest, 60(2), 290-306.

Tomkinson, G., \& Olds, T. (2007). Pediatric Fitness: Secular Trends and Geographic Variability (Vol. 50). Switzerland: Karger.

Ulrich, A. D. (2000). Test of gross motor development. Examiner's manual (2 ed.). Austin: Pro-ed.

Wedderkopp, N., Jespersen, E., Franz, C., Klakk, H., Heidemann, M., Christiansen, C., . . . Leboeuf-Yde, C. (2012). Study protocol. The Childhood Health, Activity, and Motor Performance School Study Denmark (The CHAMPS-study DK). BMC Pediatrics, 12(1), 128. doi: 10.1 186/1471-2431-12-128 World Economic Forum. (2015). Global Agenda Council on the Future of Software \& Society. Deep Shift Technology Tipping Points and Societal Impact. Deep Shift Technology Tipping Points and Societal Impact. Retrieved from Geneva Switzerland: http://www3.weforum.org/docs/WEF_GAC15_Technological_Tip- 


\section{Egitania}

s c i e $\Omega$ c i a

ping_Points_report_2015.pdf

World Health Organization. (2006). WHO Child Growth Standards: Length/ height-for-age, weight-for-age, weight-for-length, weight-for-height and body mass index-for-age: Methods and development (WHO Press ed.). Geneva, Switzerland: WHO. 